Gynäkologe 2017 · 50:742-743

DOI 10.1007/s00129-017-4126-z

Online publiziert: 17. August 2017

๑) Springer Medizin Verlag GmbH 2017

CrossMark

\author{
R. Zimmermann ${ }^{1} \cdot \mathrm{K}$. Vetter ${ }^{2}$ \\ 'Klinik für Geburtshilfe, Universitätsspital Zürich, Zürich, Schweiz \\ ${ }^{2}$ Berlin, Deutschland
}

\title{
Schwangerschaft mit 40+
}

Eudämonie - das ist das hehre Ziel, das manchen doch noch erreichbar scheint.

Eine Schwangere suchte Unterstützung bei der Geburtsplanung ihres 2. Kindes, das ihre Familie komplettieren sollte, nachdem es mit dem ersten Kind 2 Jahre zuvor gut geklappt hatte. Die Sekretärin wollte das Geburtsdatum nach der Konsultation um 10 Jahre korrigieren, die Schwangere könne doch nicht 56 Jahre alt sein. War sie aber. Sie sah vielleicht nicht so aus. Aber sie war eben vollkommen zufrieden, was sie auch nach der Geburt blieb und wohl auch heute noch diversen Jahren noch ist.

Wir glauben, dass es unsere Aufgabe ist, die Aspekte später Mutterschaft unter aktuellen Gesichtspunkten zu beleuchten. Da können wir nicht bei den neuerdings vielfach besonders bei „celebrities“ fälschlich heruntergespielten Risiken bleiben, die späte Schwangerschaften tatsächlich aber beinhalten. Im Sommer 2001 hat die Amerikanische Gesellschaft für Reproduktionsmedizin, ASRM, die Bevölkerung mit einer besonderen Kampagne aufzurütteln versucht. Die Plakate zeigten eine auf den Kopf gestellte Babyflasche in Sanduhrform. Die bereits in die untere Hälfte geflossene Milch sollte symbolisieren, dass die Zeit zum Kinder-Bekommen unaufhaltsam verrinnt. Während es die Intention dieser Poster war, auf die nach 30 rapide abnehmende Fertilität hinzuweisen, präsentiert Christian Haslinger eine aktuelle Bestandsaufnahme, die auch die zahlreichen gesundheitlichen Probleme bei später Schwangerschaft zusammenfasst. Damit ist sie nicht nur für den Kliniker nützlich.

In der 1955 erschienenen 4. Auflage seines Buches Praktische Geburtshilfe für Studierende und Ärzte definiert W. Pschyrembel: „Erstgebärende, die älter als 28-30 Jahre alt sind, sind ,alte
Erstgebärende““. Diese nicht nur medizinisch, sondern auch normativ geprägte Definition wurde über die letzten 2 Generationen von zahlreichen Fachgesellschaften und Lehrbuchautoren heraufgesetzt, zunächst auf 35 und jetzt bald auf 40 Jahre. Andernfalls würde die Pschyrembel-Definition aus dem Jahr 1955 mittlerweile die Mehrheit der erstmals Schwangeren als „alte Erstgebärende" definieren. Jürgen Dorbritz und Sabine Diabaté führen uns daher in die nicht so lineare Entwicklung der Epidemiologie später Schwangerschaften ein. Insbesondere zeigen sie, dass es parallele Entwicklungen in Österreich, der Schweiz und Deutschland - Ost wie West - gegeben hat bzw. gibt, die insbesondere durch Nachholeffekte gekennzeichnet sind, wie sie nach dem Zweiten Weltkrieg deutlich zu beobachten waren. Dorbritz und Diabaté finden dabei Hinweise auf einen möglichen Sättigungseffekt.

Als bekannt wurde, dass eine 66-jährige pensionierte Pfarrerin Zwillinge geboren hatte, titelte die Schweizer Boulevardzeitung Blick am 04.03.2012 „Ist das nicht Sünde?".Johannes Bitzerbefasst sich mit der schon in der Bibel thematisierten "greisen Schwangeren“. Dabei geht er auf die psychologischen, philosophischen, rechtlichen und moralischen Fragen der Schwangerschaft jenseits der Menopause ein. Er zeigt, dass wir es mit völlig veränderten Familienmodellen und mit Generationenverschiebungen zu tun haben, deren psychosoziale Folgen komplex sind. Insbesondere ist offen, wie all dies die Entwicklung der Kinder zu beeinflussen vermag.

Im Jahr 2015 wurde Frauen in Brasilien geraten, Schwangerschaften aufgrund der Gefahr einer Zika-Infektion um 1-2 Jahre zu verschieben. Im Mai 2017 wurde diese Empfehlung wieder ge- 
stoppt, da die Zahl von Neuinfektionen um $95 \%$ zurückgegangen war. In den 2 Jahren war der Grad der Durchseuchung mit Zika so hoch, dass ein Großteil der Bevölkerung immun und damit das Risiko einer Übertragung in der Schwangerschaft sehr klein geworden war. Genau diesen Punkt greifen Mikko Myrskylä, Kieron Barclay und Alice Goisis auf und stellen die Vorteile später Mutterschaft vor. Kinder zu einem späteren Zeitpunkt zu bekommen, hat in Zeiten neuer medizinischer Erkenntnis, wie das ZikaBeispiel zeigt, einen klaren Vorteil. Sie weisen aber auch multiple andere Veränderungen nach, die in den letzten Jahren zu Vorteilen später Mutterschaft für Mütter und Kinder geführt haben, als deren Basis Eudämonie angesehen werden kann, die anders nicht hätten erreicht werden können.

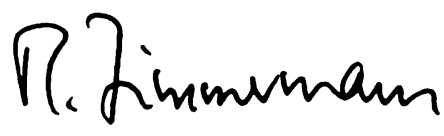

Prof. Dr. R. Zimmermann

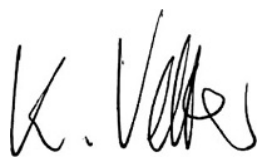

Prof. Dr. K. Vetter

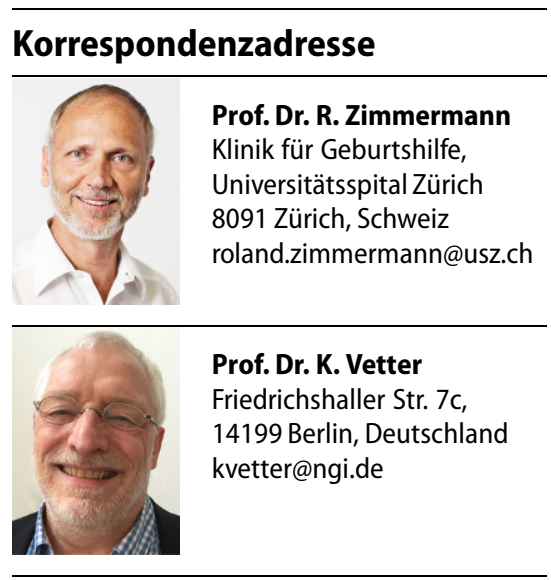

Interessenkonflikt. R. Zimmermann und K. Vetter geben an, dass kein Interessenkonflikt besteht.

\section{Bild und Fall}

Gynäkologische Diagnostik selbst verfasst

Sehr geehrte Autorin,

sehr geehrter Autor,

wir freuen uns, wenn Sie die Zeitschrift Der Gynäkologe mitgestalten. Anhand von kurzen Fallbeispielen zeigen Sie Besonderheiten der ärztlichen Praxis, Fallstricke in der Diagnostik und ungewöhnliche Krankheitsverläufe auf. Sie vermitteln wertvolle Hinweise zu Diagnostik und Therapie. Im Mittelpunkt steht Ihr Bildmaterial, anhand dessen der Leser seine Diagnose stellen und später prüfen kann, ob er richtig lag.

\section{Checkliste zur Manuskripterstellung:}

- Text bitte immer als Datei schicken (.doc)

- Komplette Anschrift der Korrespondenzadresse mit E-Mail sowie Portraitfoto

- Gesamtumfang: max. 10.000 Zeichen inkl. Leerzeichen

- Kurzer, prägnanter Beitragstitel, ggf. erläuternder Untertitel

- Englischer Titel

- Gliederung in Anamnese, Befunde, Diagnose, Verlauf und Therapie, Diskussion

- Prägnante und möglichst kurze Zwischenüberschriften

- Fazit für die Praxis (max. 500 Zeichen)

- 3-4 Abbildungen

\section{Bitte senden Sie Ihren Beitrag an:}

Prof. Dr. R. Felberbaum

ricardo.felberbaum@klinikum-kempten.de

Prof. Dr. R. Kreienberg

r.kreienberg@t-online.de

Dr. B. Ramsauer

babett.ramsauer@deidu.de

Weitere Informationen finden Sie unter www.DerGynaekologe.de
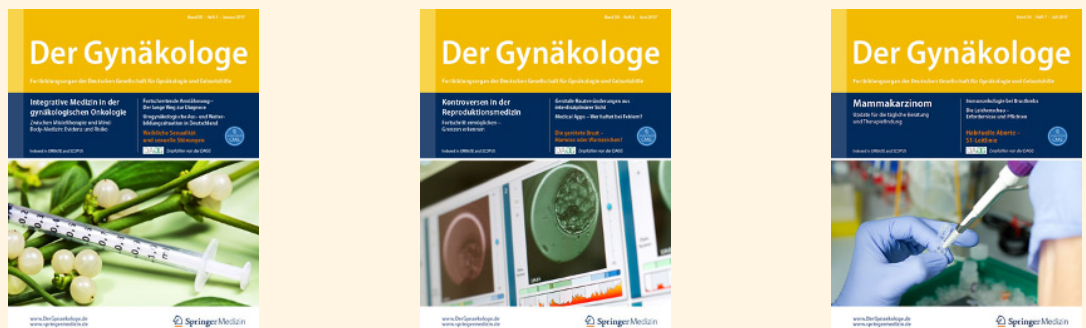